\title{
Conhecimento de mães sobre os procedimentos de emergência nos casos de avulsão dentária
}

\section{Knowledge of mothers about emergency management of tooth avulsion}

\author{
Yasmin Etienne Albuquerque* \\ Fernanda Lopez Rosell** \\ Elaine Pereira da Silva Tagliaferro ${ }^{* * *}$ \\ Silvio Rocha Corrêa da Silva ${ }^{* * * *}$
}

\section{Resumo}

Apesar de os traumatismos dentários serem frequentes na população infantil, os estudos disponíveis mostram um baixo conhecimento dos adultos sobre esse assunto. Objetivo: nesse sentido, este estudo tem por objetivo avaliar, por meio de um questionário, o conhecimento das mães que participam das atividades da Pastoral da Criança da cidade de Araraquara, SP, frente aos procedimentos de urgência nos casos de traumatismo com avulsão dentária. Materiais e método: o estudo descritivo foi realizado em uma amostra de conveniência com 65 mães que responderam a um questionário, desenvolvido para a pesquisa, composto por 15 questões sobre dados pessoais e conhecimentos sobre a avulsão dentária. Resultados: os resultados apontaram que as mães tinham em média 35 anos de idade. Cerca de 30,8\% delas relataram que seus filhos já sofreram algum tipo de traumatismo dentário. A maioria delas, (76,9\%), relatou que nunca recebeu orientações sobre esse tema e, $69,2 \%$ não sabia como os dentes são mantidos na arcada dentária. Quase metade das mães (49,2\%) acreditava que um dente avulsionado poderia ser reimplantado. Quanto ao manejo, 40\% das participantes limparia o dente avulsionado com água, mesmo que ele não estivesse sujo (38,5\%). Quanto à forma de pegar no dente para a limpeza, 69,2\% pegariam em qualquer região do dente com as mãos e acreditavam ser importante esfregar o dente para retirar toda a sujeira $(67,7 \%)$. Conclusão: com base nesses dados, conclui-se que o conhecimento das mães sobre as condutas mais apropriadas frente à avulsão dentária é inadequado, colocando em risco o sucesso do tratamento.

Palavras-chave: Avulsão dentária. Conhecimento. Criança.

\section{Introdução}

Segundo a classificação de traumatismos dentários da Organização Mundial de Saúde, a avulsão dentária é uma lesão grave que consiste na completa exarticulação do dente do seu alvéolo․ É a lesão dentoalveolar que mais causa danos funcionais, estéticos e psicológicos ao paciente, constituindo-se em uma grave urgência odontológica ${ }^{2}$. Ocorre principalmente em crianças ${ }^{3-7}$, com prevalência estimada de $1 \%$ a $16 \%$.

As consequências de um traumatismo dentário são um problema para a odontologia ${ }^{4}$ e o sucesso do tratamento da avulsão dentária depende basicamente de como a assistência de emergência é fornecida. $\mathrm{O}$ reimplante imediato é o tratamento mais apropriado para dentes permanentes traumaticamente avulsionados na tentativa de se conseguir um bom prognóstico, devendo então, ser realizado imediatamente após o acidente, se possível, ainda no local. A taxa de sobrevivência de um dente reimplantado está associada à preservação da vitalidade do seu ligamento periodontal, que por sua vez está diretamente relacionada a danos ou ao manejo inadequado da raiz (pela vítima ou pela pessoa que dá os primeiros socorros), ao tempo extra-alveolar, ao meio de armazenamento em que o dente é mantido até o reimplante (no caso de reimplante tardio) e ao tipo de reabsorção desenvolvida durante o processo de cicatrização $0^{7,9-12}$.

Mestranda, Departamento de Clínica Infantil da Faculdade de Odontologia de Aaraquara, Unesp-SP, Brasil.

Professora Doutora do Departamento de Odontologia Social da Faculdade de Odontologia de Araraquara, Unesp-SP, Brasil.

Professora Doutora do Departamento de Odontologia Social da Faculdade de Odontologia de Araraquara, Unesp-SP, Brasil.

Professor Doutor do Departamento de Odontologia Social da Faculdade de Odontologia de Araraquara, Unesp-SP, Brasil. 
Mesmo a literatura indicando a eficiência do reimplante imediato ${ }^{13}$, observa-se que esse é muito pouco realizado $0^{4,14-16}$. A maior parte dos dentes avulsionados por traumatismos é perdida e quando reimplantados, podem apresentar, a longo prazo, necrose pulpar, calcificações e reabsorções radiculares ${ }^{17}$.

Estudos $^{18-28}$ realizados em diferentes países têm demonstrado que o conhecimento, tanto de pessoas leigas como de profissionais (professores, educadores físicos, cirurgiões-dentistas e médicos) sobre traumatismos dentários é inadequado e, com isso, possíveis sequelas não são reduzidas. Um estudo realizado na Jordânia ${ }^{4}$ mostrou que $43,1 \%$ das crianças procuraram tratamento dentário apenas quando complicações tardias apareceram e o tempo entre o traumatismo e a procura por tratamento odontológico variou entre 2 horas e 3 anos.

$\mathrm{O}$ atendimento que deveria ser imediato muitas vezes não é efetivamente realizado devido à falta de conhecimento de pais e responsáveis ${ }^{29-31}$. Esse fator, associado à falta de conhecimento dos profissionais de saúde sobre os traumatismos dentários, ocasionam adiamento da avaliação pelo cirurgião-dentista $^{32,33}$, afetando o seu prognóstico.

Portanto, é muito importante envolver as pessoas (crianças, pais e professores) que podem estar presentes no local do acidente e informá-las sobre como gerenciar uma situação de traumatismo dentário.

A Pastoral da Criança ${ }^{34}$ é um organismo de ação social da Conferência Nacional dos Bispos do Brasil - CNBB, que tem como objetivo a promoção do desenvolvimento integral das crianças em seu ambiente familiar e em sua comunidade. Seus líderes atuam nas suas próprias comunidades, e por isso, conhecem as famílias e as condições em que essas vivem, podendo, assim, colaborar para a melhoria da realidade. Cada líder possui um guia de orientação para as famílias assistidas. Esse guia foi analisado e percebeu-se que não há nenhum relato de instruções sobre os procedimentos de emergência nos casos de traumatismo dentário.

Assim, este estudo foi realizado com o objetivo de avaliar o conhecimento das mães que participam das atividades da Pastoral da Criança da cidade de Araraquara, SP, frente aos procedimentos de emergência nos casos de avulsão dentária.

\section{Materiais e método}

O estudo descritivo foi realizado durante o ano de 2011 e constituiu-se da aplicação de questionários em uma amostra de mães que frequentavam as atividades da Pastoral da Criança no município de Araraquara.

Foram utilizados como critérios de inclusão, mães com 18 anos ou mais de idade que participaram das atividades da Pastoral da Criança da cidade de Araraquara e que concordaram em assinar o Termo de Consentimento Livre e Esclarecido. No to- tal, 65 das 80 mães que participaram das atividades da Pastoral da Criança durante o ano de 2011 fizeram parte da pesquisa. O estudo foi aprovado pelo Comitê de Ética em Pesquisa da Faculdade de Odontologia de Araraquara, Unesp (Protocolo n. 57/10).

A coleta dos dados foi realizada por um único pesquisador, previamente treinado, em forma de entrevista por meio de um questionário composto por 15 questões, 13 abertas e 2 fechadas, o qual foi elaborado a partir de outros estudos ${ }^{4,29}$. $\mathrm{O}$ instrumento foi dividido em duas partes: a primeira parte foi composta de variáveis sociodemográficas, com o objetivo de caracterizar as participantes por meio da idade, do estado civil, dos anos de estudo, da ocupação, da posse da residência; e a segunda abordou os procedimentos sobre a avulsão dentária, a importância do tratamento de emergência e de como agir, entre outros.

Antes da aplicação definitiva do questionário, foi realizado um pré-teste com sete mães com perfil demográfico semelhante às participantes, a fim de adequar as perguntas aos objetivos do estudo. Também se realizou o teste-reteste nas mesmas mães do pré-teste, para confirmar a reprodutibilidade do questionário por meio da aplicação do Coeficiente Kappa de acordo com os critérios propostos por Landis e $\mathrm{Koch}^{35}$ (1977). A primeira e a segunda aplicação foram realizadas pelo mesmo pesquisador e com intervalo de oito dias entre elas. A concordância Kappa foi de $87 \%$ (IC 95\%, 0,805-0,935).

As respostas das mães sobre as condutas nos casos de avulsão dentária foram avaliadas e consideradas adequadas ou inadequadas, de acordo com as orientações clínicas preconizadas por Andersson et al. ${ }^{12}$ (2012) e Barret e Keny ${ }^{5}$ (1997).

Após todos os dados terem sido coletados, as mães foram informadas sobre o assunto abordado e eventuais dúvidas foram esclarecidas.

A apuração e a análise do questionário foram realizadas por meio do programa Epi-Info versão 3.5.1. Os resultados foram expressos em tabelas com a distribuição de frequência em números absolutos e porcentuais.

\section{Resultados}

No presente estudo, 65 mães com idade média de $35( \pm 7,2)$ anos (idade mínima de 18 anos e máxima de 50 anos) preencheram o questionário. A maioria das entrevistadas estava casada $(72,3 \%)$, morava em residência própria $(70,8 \%)$ e procurava atendimento odontológico em postos de saúde $(53,8 \%)$. Quanto à escolaridade, $44,6 \%$ possuíam ensino médio completo e apenas $9,2 \%$ das mães possuíam nível superior.

Sobre a experiência prévia das mães com traumatismo dentário, 30,8\% afirmaram que seus filhos já haviam sofrido algum tipo de traumatismo dentário e apenas $23,1 \%$ já haviam recebido alguma 
instrução sobre os procedimentos mais indicados nesses casos.

A Tabela 1 mostra o conhecimento que as mães apresentavam sobre a manutenção dos dentes e seu eventual reimplante.

Tabela 1 - Distribuição das respostas das mães $(n=65)$ sobre a manutenção dos dentes na arcada dentária e reimplante dental. Araraquara, 2011

\begin{tabular}{l|c|c}
\multicolumn{1}{c|}{ Manutenção dos dentes e seu reimplante } & $\mathrm{n}$ & $\%$ \\
\hline $\begin{array}{l}\text { Conhecimento sobre como os dentes são } \\
\text { mantidos na arcada dentária }\end{array}$ & & \\
Não & 45 & 69,2 \\
Sim, pelas raízes & 20 & 30,8 \\
\hline Conhecimento sobre a possibilidade de um & & \\
dente avulsionado ser reimplantado & & \\
Sim & 32 & 49,2 \\
Não & 22 & 33,8 \\
Depende & 7 & 10,8 \\
Não sabe & 4 & 6,2 \\
\hline
\end{tabular}

$\mathrm{Na}$ Tabela 2, estão discriminadas as respostas sobre a limpeza do dente avulsionado, pelas quais se observa que, mesmo que o dente não aparentasse estar sujo, 70,8\% das mães relataram que o limpariam.

Tabela 2 - Distribuição das respostas das mães $(n=65)$ sobre as atitudes quanto à limpeza de um dente avulsionado, Araraquara, 2011

\begin{tabular}{l|r|r}
\multicolumn{1}{c|}{ Dente avulsionado } & \multicolumn{1}{c|}{$\mathrm{n}$} & \multicolumn{1}{c}{$\%$} \\
\hline Precisa ser limpo & 19 & 29,2 \\
Não & 46 & 70,8 \\
Sim & 26 & 40,0 \\
Água & 6 & 9,2 \\
Soro Fisiológico & 6 & 9,2 \\
Escova de dente & 4 & 6,2 \\
Pasta de dente & 2 & 3,1 \\
Álcool & 1 & 1,5 \\
Água e sabão & 1 & 1,5 \\
Enxaguante bucal & & \\
\hline Precisa esfregar o dente para limpar & 44 & 67,7 \\
Sim & 17 & 26,1 \\
Não & 4 & 6,2 \\
Não sabe & & \\
\hline Como pegar no dente & 45 & 69,2 \\
Em qualquer lugar com a mão & 4 & 6,2 \\
Em qualquer lugar com uma luva & 4 & 6,2 \\
Em qualquer lugar com uma pinça & 4 & 6,2 \\
Em qualquer lugar com um papel & 1,5 \\
Em qualquer lugar com um algodão & \\
ou gaze & & \\
Não sabe & & \\
\hline & & \\
\hline
\end{tabular}

A Tabela 3 mostra o conhecimento das mães sobre o reimplante do dente e o meio de transporte até o cirurgião-dentista.

Tabela 3 - Distribuição das respostas das mães $(n=65)$ sobre o reimplante dentário e a maneira de transportar o dente avulsionado. Araraquara, 2011

\begin{tabular}{l|r|r}
\hline \multicolumn{1}{c|}{ Reimplante e transporte do dente } & $\mathrm{n}$ & $\%$ \\
\hline Tempo que o dente pode ficar fora da & & \\
boca, antes de ser reimplantado & 3 & 4,6 \\
Não pode ser reimplantado & 16 & 24,6 \\
Até 4 horas & 9 & 13,9 \\
Até 1 dia & 5 & 7,7 \\
Até 1 semana & 32 & 49,2 \\
Não sabe & & \\
\hline Como deve ser o transporte do dente & & \\
avulsionado até o dentista & 16 & 24,6 \\
Recipiente limpo & 12 & 21,5 \\
Não sabe & 7 & 10,8 \\
Enrolado em um papel & 7 & 10,8 \\
Recipiente com gelo & 5 & 7,7 \\
Enrolado em um algodão & 2 & 3,1 \\
Recipiente com soro ou leite & 3,1 \\
Recipiente com álcool & & \\
Copo com água & & \\
\hline
\end{tabular}

\section{Discussão}

A falta de informação sobre a avulsão dentária por parte das pessoas que podem prestar os primeiros socorros no momento do acidente é um fator que contribui bastante para a perda prematura de um dente avulsionado, pois uma conduta realizada de maneira correta durante o pronto atendimento é crucial para o sucesso do tratamento.

Neste estudo, cerca de 30,8\% das mães que participavam das atividades da Pastoral da Criança de Araraquara, em 2011, relataram que os filhos já tinham sofrido traumatismo dentário (TD). Tais achados são semelhantes aos encontrados no Iraque $^{36}$, onde mães reportaram uma ocorrência de $38 \%$ de TD. Em estudo realizado no Piauíi ${ }^{37}$, a ocorrência de avulsões dentárias foi considerada fato comum, respondendo por quase metade (45\%) de todas as lesões traumáticas luxativas ocorridas em crianças, indicando a necessidade de atenção e de conhecimento por parte dos pais/responsáveis/cuidadores, bem como, dos profissionais de saúde.

Quando questionadas sobre a possibilidade de reimplante do dente avulsionado, 49,2\% acreditam que o reimplante pode ser realizado, porém nenhuma relatou que realmente o faria por iniciativa própria. Estudos ${ }^{12,36,38}$ revelam que uma porcentagem considerável de mães não realizaria o reimplante do dente avulsionado. A falta de conhecimento, o medo 
de prejudicar a criança e a associação percebida entre sangramento e morte faz com que os pais não tomem essa iniciativa ${ }^{39}$. Portanto, esses resultados merecem atenção pelo fato de o reimplante imediato ser o tratamento mais apropriado para tentar se obter um prognóstico favorável.

Sobre o tempo que um dente avulsionado pode permanecer fora da boca, $49,2 \%$ relataram não saber e $7,7 \%$ acreditavam que poderia permanecer por até uma semana. A maioria (57\%) das mães iraquianas $^{36}$, por sua vez, relataram ser importante a procura de um profissional dentro dos primeiros 30 minutos, em caso de avulsão dentária. De fato, a procura de ajuda profissional diante dessa situação é extremamente necessária, pois o reimplante realizado nos trinta primeiros minutos após a avulsão gera um percentual de sucesso muito elevado, porém, com o decorrer do tempo, as células do ligamento periodontal vão perdendo a vitalidade e esse percentual diminui consideravelmente ${ }^{40}$.

Sobre a condição hipotética de como o dente deve ser retirado do local do acidente, nenhuma mãe respondeu abertamente que pegaria o dente avulsionado exclusivamente pela coroa e $69,2 \%$ responderam que pegaria de qualquer forma com a mão. Resultado diferente do encontrado em outro estudo ${ }^{41} \mathrm{em}$ que apenas $10 \%$ dos participantes relataram que segurariam o dente de qualquer modo. Ao serem questionadas se realizariam a limpeza de um dente avulsionado que estivesse no o chão, 70,8\% das mães, ralataram que fariam, considerando que $67,7 \%$ delas acham importante esfregar o dente durante essa limpeza. Esses resultados corroboram os demonstrados em outros estudos $^{38,39,42}$, os quais mostraram que os pais não possuem qualquer conhecimento sobre a limpeza de dentes avulsionados. Sabe-se que a raiz de um dente avulsionado jamais deve ser esfregada, pois ali estão localizados os remanescentes periodontais que são de fundamental importância na reinserção dos dentes reimplantados ${ }^{7}$. Portanto, deve-se evitar esfregar ou simplesmente tocar no dente pela raiz, pois, além de injuriar os remanescentes periodontais, essa prática aumenta também o nível de contaminação do dente avulsionado. Se o reimplante imediato não for possível, o dente deve ser manipulado somente pela coroa, para que o ligamento periodontal não seja danificado, devendo ser armazenado imediatamente em meio úmido para preservar as células do ligamento periodontal que são fundamentais para possibilitar a revascularização até se chegar a um cirurgião-dentista ${ }^{12}$. Neste estudo, o transporte do dente até o dentista seria feito, por $24,6 \%$ das mães, em recipiente limpo e apenas $7,7 \%$ o transportariam em um recipiente com soro fisiológico ou leite. Resultados abaixo do encontrado em outro estudo ${ }^{18}$ que reportou que $66,5 \%$ colocariam o dente de volta na boca da criança ou transportariam o mesmo até o dentista e 25,6\% armazena- riam o dente avulsionado em recipiente com soro fisiológico ou leite. Em outro estudo ${ }^{43}$, os autores obtiveram $21,5 \%$ de respostas em que o leite foi o escolhido como meio de armazenamento e 58,3\% dos participantes do estudo disseram não saber qual seria o meio de transporte ideal. De fato, os dentes acondicionados em meio seco perdem rapidamente a vitalidade do ligamento periodontal e, dessa forma, obrigatoriamente deve-se armazená-lo em meio aquoso ou saliva ${ }^{44}$.

De acordo com a literatura ${ }^{45}$, não existe um único produto ou solução que possui todas as características necessárias para ser indicado como o meio de armazenamento ideal para dentes avulsionados. No momento, o meio mais indicado é a solução salina balanceada de Hanks ${ }^{\circledR}$, por melhorar a sobrevivência das células periodontais da superfície radicular e ajudar a remover os debris da raiz e dissolver bactérias. O leite bovino é considerado um meio de conservação alternativo por ser o meio mais fácil de ser encontrado no local ou próximo ao acidente, possuir pH e osmolaridade compatíveis aos das células vitais e ser relativamente livre de bactérias ${ }^{12}$. Estudos mostraram que o leite bovino pode manter a efetividade das células do periodonto por um período que varia de 3 a 6 horas ${ }^{5,17}$. A saliva mantém o dente úmido, porém, não é considerado um meio de armazenamento ideal devido a osmolaridade e $\mathrm{pH}$ incompatíveis, além da presença de bactérias, portanto, só deve ser utilizado como última opção ${ }^{12}$.

De acordo com o Guia de Avaliação e Manejo de Trauma Dentário da Associação Internacional de Traumatologia Dentária (AITD), divulgado em 2012, a recomendação atual para o transporte de um dente avulsionado é evitar o armazenamento em água e utilizar a solução salina balanceada de Hanks $^{\circledast}$ ou o leite. Caso nenhuma das duas opções esteja disponível, o transporte deve ser realizado no interior do lábio ou bochecha da boca do paciente ${ }^{12}$. De fato, um estudo demonstrou ${ }^{46}$ que pacientes que sofreram algum tipo de traumatismo dentário, e foram tratados seguindo as recomendações da AITD, obtiveram menores taxas de complicações pós-trauma, se comparados aos pacientes que foram tratados sem tais orientações.

De forma geral, os achados deste estudo também evidenciaram que nenhuma das mães demonstrou possuir conhecimento suficiente sobre o assunto. Observou-se no estudo que as mães são carentes inclusive de informações básicas, pois ao serem questionadas sobre como os dentes são mantidos na arcada dentária, $69,2 \%$ das participantes responderam não saber. Ainda, quando as mães foram questionadas se eles receberam previamente qualquer informação sobre o assunto, 76,9\% delas relataram nunca terem recebido qualquer orientação sobre o assunto. Esse resultado corrobora aos encontrados em outros estudos ${ }^{4,36,39,47}$.

Portanto, considerando o fato de que os pais podem desempenhar um papel importante no prog- 
nóstico de dentes avulsionados, há necessidade da realização de campanhas educativas para a população leiga em geral. A falta desse conhecimento pode gerar complicações físicas, estéticas e emocionais, bem como os altos custos dos tratamentos que precisam ser realizados nesse caso. Já há estudos ${ }^{48-51}$ que evidenciaram mudança de comportamento e grau de conhecimento de profissionais de Educação, após atividades educativas sobre o assunto. Vale salientar que, embora haja limitações na transmissão da mensagem para um entendimento completo, um folheto simples com informações básicas importantes é capaz de elevar o nível de conhecimento dos pais de $46 \%$ para $74 \%{ }^{52}$. Outro estudo ${ }^{53}$ sugere a realização de consultas odontológicas de rotina como um veículo educacional para transmitir informação sobre a gestão em casos de traumatismos dentários. É importante enfatizar a necessidade de se procurar um especialista, pois, de acordo com um estudo recente, os cursos de especialização são importantes para a atuação correta e efetiva dos cirurgiões-dentistas nos casos de traumatismos dentários ${ }^{54}$. Um terceiro estudo sugere a inclusão de gestão de dentes traumatizados como um tópico na grade curricular de universidades ${ }^{47}$. Assim, campanhas preventivas devem ser realizadas, além da necessidade de inclusão de informações sobre o tema no guia de orientações dos líderes da Pastoral da Criança, na tentativa de se conseguir um bom prognóstico do tratamento e aumentar a taxa de sobrevivência dos dentes reimplantados.

Por fim, é importante mencionar as limitações deste estudo, como a obtenção de informação sobre eventos que ocorreram no passado, que pode estar sujeito ao viés de memória, bem como, a se deve considerar a validade externa dos dados, uma vez que os resultados são válidos apenas para esse grupo de mães que participavam das atividades da Pastoral da Criança da cidade de Araraquara, no ano de 2011, não podendo ser generalizados para todas as mães do município.

\section{Conclusão}

Os resultados encontrados nesse estudo indicam que o conhecimento das mães que participam das atividades da Pastoral da Criança da cidade de Araraquara, a respeito dos procedimentos de emergência em caso de avulsão dentária é inadequado, em vários aspectos. Isso indica a urgente necessidade de acesso à informação para que o manejo inicial em casos de avulsão seja adequado, evitando, assim, o comprometimento do prognóstico e possível sucesso do tratamento dessas lesões.

\section{Abstract}

Introduction: Despite tooth avulsion following trauma being relatively common in children, the available studies show that adults have limited knowledge about it. Aim: The purpose of this study was to assess, by a questionnaire, the mothers' general knowledge about the immediate management of tooth avulsion. Material and method: This descriptive study was carried out on a convenient sample of mothers $(n=65)$ who participated of the "Pastoral da Criança", from Araraquara, SP, Brazil. The questionnaire comprised 15 questions about personal data and knowledge on tooth avulsion management. Results: Participants were, on average, 35 years old. A total of $30.8 \%$ of the mothers reported that their children suffered dental trauma. The majority had never received advice on this subject (76.9\%); and did not know how teeth are kept in the dental arch (69.2\%). Almost a half of the sample believed that an avulsed tooth can be replanted (49.2\%). In relation to the management of tooth avulsion, $40 \%$ of them would clean the avulsed tooth with water, even if it was not dirty (38.5\%). Most of them (69.2\%) would take the tooth by hand for cleaning purposes, regardless the tooth region; and believed that brushing the tooth was important to take the dirty out (67.7\%). Conclusion: The general knowledge of mothers about the immediate management of tooth avulsion was considered inadequate endangering the successful treatment of tooth avulsion.

Keywords: Tooth avulsion. Knowledge. Child.

\section{Referências}

1. Guedes OA, Alencar AHG, Lopes LG, et al. A retrospective study of Traumatic Dental Injuries in a Brazilian dental urgency service. Braz Dent J 2010; 21(2): 153-7.

2. Moreira Neto JJS, Gondim JO, Carvalho FM, Giro EM. Longitudinal clinical and radiographic evaluation of severely intruded permanent incisors in a pediatric population. Dent Traumatol 2009; 25(5): 510-4.

3. Perri de Carvalho, AC. Reimplante dental imediato. Relato de campanha de esclarecimento. Rev Assoc Paul Cirurg Dent. 1988; 42(3): 248-9.

4. Al-Jundi SH. Knowlegde of Jordanian Mothers with regards to emergency management of dental trauma. Dent Traumatol. 2006; 22(6): 291-5.

5. Barrett EJ, Kenny DJ. Avulsed permanent teeth: a review of the literature and treatment guidelines. Endod Dent Traumatol. 1997; 13(4): 153-63.

6. Glendor U. On dental trauma in children and adolescents. Incidence, risk, treatment, time and costs. Swed Dent J Suppl. 2000; 140: 1-52.

7. Vasconcelos BCE, Laureano Filho JR, Fernandes BC, Aguiar ERB. Reimplante dental. Rev Cir Traumat Buco-Maxilo-Facial. 2001 ; $1(2)$ : 45-51.

8. Flores MT, Andersson L, Andreasen JO, Bakland LK, Bourguignon C, Diangelis A, et al. Guidelines for management of traumatic dental injuries. II. Avulsion of permanente teeth. Dent Traumatol. 2007; 23(3): 130-6.

9. Andreasen JO, Andreasen FM, Andersson L. Textbook and color atlas of traumatic injuries to the teeth, 4th ed. Oxford: Wiley-Blackwell; 2007, 912 p. 
10. Andreasen JO, Hjørting-Hansen E. Replantation of teeth. I. Radiographic and clinical study of 110 human teeth replanted after accidental loss. Acta Odontol Scand. 1966; 24(3): 263-86.

11. Blomlöf L, Andersson L, Lindskog S, Hedström KG, Hammarström L. Periodontal healing of replanted monkey teeth prevented from drying. Acta Odontol Scand. 1983; 41(2): 117-23.

12. Andersson L, Andreasen JO, Day P, Heithersay G, Trope M, Diangelis AJ, KenNy DJ, Sigurdsson A, Bourquignon C, Flores MT, Hicks ML, Lenzi AL, Malmgren B, Moule AJ, Tsukiboshi M. International Associantion of Dental Traumatology guidelines for the management of traumatic dental injuries: 2. avulsion of permanent teeth. Dent Traumatol. 2012; 28(2): 88-96.

13. Andreasen, J. O. Atlas of replantation and transplantation of teeth. Switzerland: Ed. Med Globe; 1992.

14. Oliveria TM, Sakai VT, Moretti AB et al. Knowledge and attitude of mothers with regards to emergency management of dental avulsion. J Dent Child 2007; 74(3): 200-2.

15. Trope M. Clinical management of the avulsed tooth. Dent Clin North Am. 1995; 39(1): 93-112.

16. Waldon K, Barber SK, Spencer RJ, Duggal MS. Indications for the use of auto-transplantation of teeth in the child and adolescent. Eur Arch of Paediatr Dent. 2012; 13(4): 210-6.

17. Bittencourt AM, Pessoa OF, Silva JM. Avaliação do conhecimento de professores em relação ao manejo da avulsão dentária em crianças. Rev Odontol Unesp. 2008; 37(1): 15-9.

18. Hegde AM, Kumar KNP, Varghese E. Knowledge of dental trauma among mothers in Mangalore. Dent Traumatol. 2010; 26(6): 325-9.

19. Hashim R. Investigation of mothers' knowledge of dental trauma management in United Arab Emirates. Eur Arch Paediatr Dent. 2012; 13(2): 83-6.

20. Yassen GH, Chin JR, Younus MS, Eckert GJ. Knowledge and attitude of dental trauma among mothers in Iraq. Eur Arch Paediatr Dent. 2013; 14: 259-65.

21. Skeie MS, Audestad E, Bardsen A. Traumatic dental injuries - Knowledge and awareness among present and prospective teachers in selected urban and rural areas of Norway. Dent Traumatol. 2010; 26(3): 243-7.

22. Curylofo PA, Lorencetti KT, Silva SRC. Avaliação do conhecimento de professores sobre avulsão dentária. Arq Odontol. 2012; 48(3): 175-80.

23. Jorge KO, Ramos-Jorge ML, Toledo FF, Alves LC, Paiva SM, Zarzar PM. Knowledge of teachers and students in physical education's faculties regarding first-aid measures for tooth avulsion and replantation. Dental Traumatol. 2009; 25: 494-9.

24. Vergotine RJ, Govoni R. Public school educator's knowledge of initial management of dental trauma. Dent Traumatol. 2010; 26(2): 133-6.

25. Baginska J, Wilczynska-Borawska M. Continuing dental education in the treatment of dental avulsion: Polish dentists' knowledge of the current IADT guidelines. Eur J Dent Educ. 2013; 17(1): e88-e92.

26. Upadhyay S, Rokaya D, Upadhayaya. Knowledge of emergency management of avulsed teeth among general dentists in Kathmandu. Kathmandu Univ Med J. 2012; 10(38): 37-40.

27. Qazi SR, Nazir KS. First-aid knowledge about tooth avulsion among dentists, doctors and lay people. Dent Traumatol. 2009; 25(3): 295-9

28. Ulusoy AT, Onder H, Cetin B, Kaya S. Knowledge of medical hospital emergency physicians about the first-aid manage- ment of traumatic tooth avulsion. Int J Paediatr Dent. 2012; 22(3): 211-6.

29. Campos MI, Henriques KA, Campos CN. Nível de informação sobre a conduta de urgência frente ao traumatismo dental com avulsão. Pesq Bras Odontoped Clin Integr. 2006; 6(2): $155-9$.

30. Granville-Garcia AF, Menezes VA, Lira PIC. Prevalência e fatores sociodemográficos associados ao traumatismo dentário em pré-escolares. Odontol Clin-Cient. 2006; 5(1): 57-64.

31. Yassen GH, Chin JR, Al-Rawi BA et al. Traumatic Injuries of permanent teeth among 6-12 year-old Iraqi children: A 4 year retrospective study. J Dent Child (Chic). 2013; 80(1): 3-8.

32. Hamilton FA, Hill FJ, Mackie IC. Investigation of lay knowledge of the management of avulsed permanent incisors. Endod Dent Traumatol. 1997; 13(1): 19-23.

33. McIntyre JD, Lee JY, Trope M, Vann WF Jr. Elementary school staff knowledge about dental injuries. Dent Traumatol. 2008; 24(3): 289-98.

34. Pastoral da Criança. A partilha que salva vidas [Internet]. [acesso em 2011 nov 11]. Disponível em: https://www.pastoraldacrianca.org.br/index.php?option=com_content\&view $=\mathrm{a}$ rticle\&id=75:partilha-que-salva- idas\&catid=45:instituciona $1 \&$ Itemid $=27$.

35. Landis JR, Koch GG. The measurement of observer agreement for categorical data. Biometrics. 1977; 33(1): 159-74.

36. Yassen GH, Chin JR, Younus MS. Knowledge and attitude of dental trauma among mothers in Iraq. Eur Arch Paediatr Dent. 2013; 14(4): 259-65.

37. Moura LFA, Ferreira DLA, Melo CP, Sady MCLM, Moura MS, Mendes RF, Moura WL, et al. Prevalência de injúrias traumáticas em crianças assistidas na clínica odontológica infantil da Universidade Federal do Piauí, Brasil. Pesq Bras Odontop Clín Integr. 2008; 8(3): 341-5.

38. Ozer S, Yilmaz EI, Bayrak S, Tunc ES. Parental knowledge and attitudes regarding the emergency treatment of avulsed permanent teeth. Eur J Dent. 2012; 6(4): 370-5.

39. Loo TJ, Gurunathan D1, Somasundaram S. Knowledge and attitude of parents with regard to avulsed permanent tooth of their children and their emergency management-Chennai. J Indian Soc Pedod Prev Dent. 2014; 32(2): 97-107.

40. Trope M. Clinical management of the avulsed tooth: present strategies and future directions. Dent Traumatol. 2002; 18(1): 1-11.

41. Pacheco LF, Filho PF, Letra A, Menezes R, Villoria GE, Ferreira SM. Evaluation of the knowledge of the treatment of avulsions in elementary school teachers in Rio de Janeiro, Brazil. Dent Traumatol. 2003; 19(2): 76-8.

42. Al-Jame Q, Andersson L, Al-Asfour. Kuwaiti Parents' Knowledge of First-Aid Measures of Avulsion and Replantation of Teeth. Med Princ Pract. 2007; 16: 274-79.

43. Mohandas U, Chandan GD. Knowledge, attitude and practice in emergency management of dental injury among physical education teachers: a survey in Bangalore urban schools. J Indian Soc Pedod Prev Dent. 2009; 27(4): 242-8.

44. Araujo TPB, Nogueira LLA, Carvalho FP, Gomes IL, Souza SFC. Avaliação do conhecimento de pais e educadores de escolas públicas do município de São Luis, MA, sobre avulsão dental. Pesq Bras Odontoped Clin Integr. 2010; 10(3): 371-6.

45. Poi WR, Sonoda CK, Martins CM, Melo ME, Pellizzer EP, Mendonça MR, Panzarini SR. Storage media for avulsed teeth: a literature review. Braz Dent J. 2013; 24(5): 437-45.

46. Bucher K, Neuman C, Hickel R, Kühnisch J. Complications and survival of teeth after trauma over a 5-year-period. Clin Oral Investig. 2013; 17(5): 1311-8. 
47. Emerich K, Wlodarczyk P, Ziolkowski A. Education of Sport University students regarding first-aid procedures after dental trauma. Eur J Paediatr Dent. 2013; 14(1): 37-41.

48. Al-Asfour A, Andersson L, Al-Jame Q. School teachers' knowledge of tooth avulsion and dental first aid before and after receiving information about avulsed teeth and replantation. Dent Traumatol. 2008; 24(1): 43-9.

49. Poi WR, Salineiro SL, Miziara FV, Miziara EV. A educação como forma de favorecer o prognóstico do reimplante dental. Rev Assoc Paul Cirur Dent. 1999; 53(6): 474-9.

50. Frujeri MLV. Avulsão dentária: efeito da informação na mudança de comportamento em diferentes grupos profissionais. [Dissertação]. Brasília: Faculdade de Ciências da Saúde; 2006. $177 \mathrm{p}$.

51. Ghaderi F, Adl A, Ranjbar Z. Effect of a leaflet given to parents on knowledge of tooth avulsion. Eur J Paediatr Dent. 2013; 14(1): 13-6.

52. Al-Asfour A, Andersson L. The effect of a leaflet given to parents for first aid measures after tooth avulsion. Dent Traumatol. 2008; 24(5): 515-21.

53. Vergotine RJ, Koerber A. The relationship of dental visits to parental knowledge of management of dental trauma. Pediatr Dent. 2010; 32(4): 329-32.

54. Pinheiro SAA, DelfinoII CS. Conhecimento do cirurgião-dentista sobre trauma dentário. Rev. Cir. Traumatol. Buco-Maxilo-Fac. 2014; 14(1): 83-90.

\section{Endereço para correspondência:}

Silvio Rocha Corrêa da Silva

Rua Humaitá, 1680

14801-903 Araraquara-SP

Fone: (16) 3301-6355

E-mail: silvio@foar.unesp.br

Recebido: 14/10/2013. Aceito: 10/08/2014. 